\title{
A Placebo-Controlled Study Demonstrates the Long-Lasting Anti-Aging Benefits of a Cream Containing Retinol, DihydroxyMethylChromone (DMC) and Hyaluronic Acid
}

\author{
Thierry Oddos ${ }^{1}$, Romain Roure ${ }^{2}$, James Leyden ${ }^{3}$, Valérie Bruère ${ }^{2}$, Christiane Bertin ${ }^{2}$ \\ ${ }^{1}$ Johnson \& Johnson Santé Beauté France, Val-de-Reuil, France; ${ }^{2}$ Johnson \& Johnson Santé Beauté France, Issy-les-Moulineaux, \\ France; ${ }^{3}$ KGL Inc., Broomall, USA. \\ Email: toddos@its.jnj.com, rroure@its.jnj.com
}

Received February $7^{\text {th }}, 2012$; revised March $11^{\text {th }}, 2012$; accepted March $21^{\text {st }}, 2012$

\begin{abstract}
Retinol is an ingredient used in cosmetic products for reducing the appearance of the signs of aging and photo-damage. Currently, most of these products contain $0.1 \%$ of retinol. However, at this concentration, some irritation can occur. We have evaluated in vitro and in a clinical study the potential efficacy of a combination of actives to improve the facial skin aging signs while using low concentration of retinol. We demonstrated, in vitro, that a chromone derivative, 5,7di-hydroxy-2-methyl chromone (DMC), is able to enhance the collagen synthesis in culture of normal human dermal fibroblasts. The enhancement of retinol anti-wrinkle efficacy by DMC was confirmed in a small scale clinical trial. Specifically, a product associating low concentration of retinol $(0.04 \%)$ and DMC $(0.1 \%)$ in combination with low molecular weight hyaluronic acid fragments (50,000 Dalton of average molecular weight) has been applied topically for 8 weeks. Clinical results show significant improvement of various signs of facial skin aging such as wrinkles, pigmentary spots, tone unevenness, dullness and the overall photo-damage score. Improvements were still visible 4 weeks after the cessation of the test product application. This study demonstrates that significant lasting improvement of facial skin aging can be obtained with well tolerated low concentration of retinol when adequately formulated with other anti-aging ingredients.
\end{abstract}

Keywords: Retinol; DihydroxyMethylChromone; Hyaluronic Acid; Anti-Age; Long-Lasting Effect

\section{Introduction}

Clinically, facial skin aging is characterized by multiple features including fine lines, wrinkles, mottled hyper pigmentation, brown spots, laxity and loss of firmness. Repeated exposure to solar ultraviolet radiations induces skin aging signs such as deep wrinkles, loss of firmness and pigmentary changes [1-4]. These signs of chronological aging and photo-damage are associated with a general slow-down of cell renewal in the epidermis and loss of collagen synthesis in the dermis [5-9]. It was demonstrated that exposure of skin cells to sunlight radiation is associated with subclinical inflammation that induces release of degrading enzymes such as collagenase (Matrix Metalloproteinase-1, MMP1) and gelatinase (Matrix Metalloproteinase-9, MMP9) that degrades collagen in the dermis [10-12], and with the synthesis and accumulation of non functional elastin [13-15]. In the epidermis, chronological and photo aging lead to an atrophy of the epidermis with a loss of the micro relief as well as an irregular distribution of melanin [16-18].

Prevention and reduction of the appearance of the signs of aging on facial skin has been one of the main challenges in the cosmetic industry. Several categories of solutions, based on the use of active ingredients, have been developed. Among them, retinoids, a family of compounds derived from vitamin A, or molecules which interact with retinoid receptors and produce similar biological effects, decrease the severity of skin aging [1922]. Retinoids counteract some of the physiological causes leading to the appearance of skin aging signs. For example, retinoids stimulate epidermal cells proliferation through the release of Heparin Binding-Epidermal Growth Factor (HB-EGF), and inducing epidermal thickening [23-25]. They also enhance collagen synthesis and functional elastin fibers accumulation in the dermis while they prevent the over-expression of matrix metalloproteinase through the interference with the jun/fos AP1 inflammation pathway $[11,26]$. However, the cosmetic use of retinoids is limited due to their potential to cause 
skin irritation [27-29].

Hyaluronic Acid (HA) is a major component of the extracellular matrix of the dermis. It is a high-molecularweight glycosaminoglycan composed of glucuronic acid and $\mathrm{N}$-acetylglucosamine disaccharide repeats. It is known to bind water, and it has been used for decades as a moisturizing ingredient in cosmetic products. Recently, it has been shown that HA could enhance keratinocyte proliferation through CD44 receptor stimulation. Moreover, CD44 expression is reduced with exposure to UV light. This reduced expression of CD44 can be reversed by retinoids such retinol or retinaldehyde [30,31].

In this article, we describe the combination of retinol with Di-hydroxy Methyl Chromone (DMC), an active ingredient that, in our in vitro screening, showed additive effect on retinol-induced gene expression in human keratinocyte, and with low molecular weight HA, to deliver benefit on multiple signs of skin aging without significant signs of irritation. The beneficial effects of this combination of ingredients were evidenced in a double-blind placebo-controlled two-month clinical study. The results of the study show a remaining effect during the first 4 weeks after the cessation of the treatment.

\section{Materials and Methods}

\subsection{Proof of Principle}

\subsubsection{In Vitro Studies}

\subsubsection{Keratinocyte Culture}

Keratinocytes (Lonza, Verviers Belgium) were seeded at 10,000 cells per well in six wells plate and cultivated in KBM2 medium (Lonza, Verviers, Belgium) supplemented with Bovine Pituitary extract, human Epidermal Growth Factor, insulin, hydrocortisone, transferrin and epinephrine (Lonza) and antibiotics. Test ingredients were introduced in the medium and keratinocyte cultures were incubated during 48 hours at $37^{\circ} \mathrm{C}$ in a water saturated atmosphere containing $5 \% \mathrm{CO}_{2}$. Then cells were detached from plastic by trypsination. After several rinsing in phosphate buffer saline ( $\mathrm{pH}$ 7.2), cells were counted and then pelleted in microtube. RNA were extracted using a RNeasy Plus mini kit (Qiagen, Courtaboeuf, France) and the concentration of total RNA was determined by measuring the optical density at $260 \mathrm{~nm}$. The purity of the RNAs was assessed by measuring A260/A230 and A260/ A280 ratios.

\subsubsection{Analysis of Gene Expression by Real Time} Quantitative Polymerase Chain Reaction (QPCR)

$1 \mu \mathrm{g}$ of total RNA was reverse transcribed to generate first-strand cDNA using ImProm-II Reverse Transcription system (Promega, Charbonnnières, France) with random hexamers as suggested by the manufacturer instructtions. As controls, parallel reactions were run in the absence of reverse transcriptase or in the absence of input RNA to assess any genomic DNA contamination. Realtime QPCR amplification was carried out using the Brilliant SYBR Green QPCR Mix (Stratagene, Amsterdam, Netherlands) in Mx3000p detection system (Stratagene). Each sample was analyzed in duplicate along with standard and no-template controls. PCR parameters were $95^{\circ} \mathrm{C}$ for 10 minutes, 40 cycles at $95^{\circ} \mathrm{C}$ for $15 \mathrm{sec}, 60^{\circ} \mathrm{C}$ for 1 minute and $72^{\circ} \mathrm{C}$ for $30 \mathrm{sec}$. RNA concentrations were determined by determining Cycle Threshold (CT) for each sample and subsequently using the 2- $\Delta \Delta C T$ method. RNA levels were further corrected with the $18 \mathrm{~S}$ gene cDNA signal for variations in amounts of input RNA. Statistical analysis was done with the Student $t$-test $(\mathrm{p} \leq 0.05)$.

\subsubsection{Fibroblasts Culture and Collagen Synthesis Assay}

50,000 fibroblasts isolated from human abdominal skin biopsies were seeded in 24 wells multiplate in DMEM (Invitrogen, Cergy Pontoise, France) with $10 \%$ fetal calf serum (FCS) (ATGC, Marne la Vallée France) with antibiotics and incubated at $37^{\circ} \mathrm{C}$ in a humidified atmosphere. 72 hours later supernatants were replaced with DMEM containing $2 \% \mathrm{FCS}, 50 \mu \mathrm{g} / \mathrm{ml} \beta$-aminopropionitrile, 50 $\mu \mathrm{g} / \mathrm{ml}$ ascorbic acid, 3 - $4 \mu \mathrm{g} / \mathrm{ml} \alpha$-ketoglutarate (SigmaAldrich, Saint Quentin Fallavier, France) and the test product. Half of the wells $(n=4)$ received also $5 \mu \mathrm{Ci}$ of tritiated proline (Amersham-GE Healthcare, Orsay, France) for synthesis rate incorporation. The other half $(n=4)$ was treated identically with the exception of radioactive proline. These cells were counted to assess the number of cells per well. 72 hours later, supernatants were recovered and precipitated with $20 \%$ trichloro-acetic acid (TCA) (Sigma-Aldrich). Pellet were rinsed twice with 5\% TCA and dissolved in sodium hydroxide $0.05 \mathrm{~N}$. Protein solutions were treated with purified collagenase Type III (Sigma-Aldrich (Unit/ml) and reaction was stopped by precipitating the proteins with TCA $15 \%$ and tannic acid $0.75 \%$. Then the radioactivity contained in the supernatant corresponding to the collagen fraction was counted on a $\beta$-scintillation counter (Perkin Elmer, Courtaboeuf, France). Results were expressed as dpm in collagenic fraction per 1000 cells and percentage of stimulation was calculated as followed:

$$
\% \text { of stimulation }=[(\text { synthesis rate in sample }- \text { synthesis rate in control }) /(\text { synthesis rate in control })] \times 100 .
$$


Statistical analysis was done with the Student $t$-test $(\mathrm{p} \leq$ $0.05)$.

\subsubsection{In Vivo Proof of Principle}

A double blind, randomized clinical study was performed to demonstrate the additive efficacy of DMC when combined with 2 different percentages of retinol. The study was conducted from March to June of 2006.

The subjects applied the products once a day, in the morning, for 12 weeks. Products were applied ad libitum, randomly, to a split face. The three test products were randomly distributed between subjects according to a split face mode.

\subsubsection{Subjects}

34 subjects aged from 42 to 59 years old, with skin phototypes I-IV according to Fitzpatrick's classification, were involved in this study. The volunteers were in good health without any skin condition. Each volunteer signed an informed consent form before participating to the study. Two subjects withdrew from the study due to cutaneous reactions to the products.

\subsubsection{Products}

The products contained a combination of active ingredients: retinol, hyaluronic acid and DihydroxyMethylChromone (DMC). The three test products contained either $0.04 \%$ retinol or $0.1 \%$ retinol or $0.04 \%$ retinol and $0.1 \%$ DMC.

\subsubsection{Measurements}

A clinical assessment of the wrinkles was performed at baseline (T0) and after 12 weeks (T12) of products use, using a $12 \mathrm{~cm}$-visual analog scale by an expert grader. The results are given as percentage of improvement from baseline. For the comparison between products, the statistical analysis was performed on the differences between T12 and T0. A paired Student's $t$-test was done to compare the individual scores at each follow-up session relative to their respective baselines for each treatment.

\subsection{Clinical Study}

A double blind, randomized, placebo-controlled study was performed to assess the efficacy of a formula containing a combination of three active ingredients: retinol $0.04 \%$, DMC $0.1 \%$ and HA $0.1 \%$, with a special focus on the immediate and lasting effect on signs of facial photodamage. The study was conducted from March to July of 2007.

Randomization was performed by the sponsor and neither the investigator nor the volunteers knew which product was used. The investigator followed the general guidelines recommended in Good Clinical Practices (GCP) and Good Laboratory Practices (GLP).

There were 2 parallel groups, each panelist applying one product (active or placebo) on face and neck, according to the randomization. One month before the beginning of the study, all volunteers had to stop using any anti-aging products and applied, in the evening, a basic moisturizing cream that they continued to apply for the total duration of the study. During the 8-week application period, the panelists applied the product (active or placebo); once a day, in the morning. Both products were applied ad libitum. After the eight weeks of product application, all the volunteers kept using the moisturizing cream for 4 weeks (regression period). A sample daily diary form was provided to each subject. They indicated the time of product application during the entire study. Subjects were evaluated 4 weeks after discontinuation of treatment to determine if changes persisted. Prolonged exposure to the sun had to be avoided during the entire study including the preconditioning phase. If prolonged sun exposure was unavoidable, subjects were required to use a SPF $50+$ sunscreen provided by the sponsor.

\subsubsection{Subjects}

87 (eighty seven) healthy women volunteers were recruited from a pool of women who meet the inclusion criteria. To be included in the study, the subjects had to be between 35 to 60 years of age $(\mathrm{min}=37$ y.o. and $\max$ $=60$ y.o.) and had to present signs of photodamage (score superior or equal to 4 at baseline on a 10-point scale). All volunteers signed an informed consent. Two subjects withdrew for personal reasons unrelated to the study. The population was split into two groups, 44 (forty four) volunteers applied the product and 43 (forty three) the placebo, during the 8 -week treatment period.

\subsubsection{Products}

The active product contained a combination of active ingredients: retinol, hyaluronic acid and DihydroxyMethylChromone (DMC). The placebo, a basic moisturizer, was an oil in water emulsion without the active ingredients.

\subsubsection{Measurements}

The measurements employed were clinical grading of aging signs and digital photography. These measurements were performed at baseline (T0), then after four weeks (T4) and eight weeks (T8) of daily application as well as after the regression period (T12) to assess the residual effect.

More specifically, at each time point, the expert dermatologist examined the panelists and graded their face for a series of attributes: forehead wrinkles, crow's feet wrinkles, crow's feet fine lines, under eye wrinkles, cheek wrinkles, brown spots, lack of skin texture, lack of 
skin radiance, lack of evenness of skin tone, lack of skin softness, lack of skin smoothness and overall photodamage. The investigator used a 10 points structured scale with the following severity ranking: $0=$ none; 1 to $3=$ mild; 4 to $6=$ moderate and 7 to $9=$ severe.

Also, digital photographies of frontal/side shot of the face were taken using standardized equipment and procedures provided by the Sponsor. The panelists were asked to present a neutral face expression with eyes open when imaging under visible light and eyes closed for other types of light.

\subsubsection{Statistical Analysis}

The data set was analyzed by comparing the changes that occurred within each treatment group, from Baseline to each consequent timepoint. Normality of sample distribution was verified using the one-sample KolmogorovSmirnov test. According to this test, a paired Student's $t$-test (normal distribution) or a Wilcoxon signed rank test was done to compare the individual scores at each follow-up session relative to their respective Baseline for each treatment.

In addition, groups were compared based on the net change from Baseline. Normality of distribution (onesample Kolmogorov-Smirnov test) and variance equality (F-test) were verified. According to these previous tests, comparisons were done using the adequate test (either Independent Student's $t$-test with equal or unequal variance for normal distribution or Wilcoxon rank sum test).

Throughout the analysis, unless otherwise specified, a two tailed $p \leq 0.05$ was taken as the level of significance. The values reported in the results are expressed as arithmetic mean \pm standard error of the mean (SEM). All statistical tests were performed using the software Matlab ${ }^{\circledR}$.

\section{Results}

\subsection{Preliminary Assessment of the Anti Aging Efficacy of Retinol and DMC Combination}

\subsubsection{In Vitro}

The effects of DMC were assessed using different end points. Treatment of human dermal fibroblasts in culture by DMC induced a significantly higher level of collagen synthesis compared to non treated control, as measured by the incorporation of tritiated proline in the extra cellular collagenic fraction (Table 1(a)). The stimulation obtained with DMC $0.1 \mu \mathrm{g} / \mathrm{ml}$ and $1 \mu \mathrm{g} / \mathrm{ml}(46 \pm 2.2$ $\mathrm{dpm} / 10^{3}$ cells and $46.2 \pm 5.8 \mathrm{dpm} / 10^{3}$ cells respectively) were similar to the one obtained with tumor growth factor $\beta$-1 (TGF $\beta 1)\left(46.5 \pm 0.5 \mathrm{dpm} / 10^{3}\right.$ cells $)$ and significantly higher than the non treated control $(34.7 \pm 4.1$ $\mathrm{dpm} / 10^{3}$ cells ).
When tested in association with retinol in skin explants model, DMC slightly increased the steady state level of retinol-induced HB-EGF and CRABP2 mRNA, two markers of retinol activity in epidermis of human skin explants, although this effect was not statistically significant (Table 1(b)).

\subsubsection{In Vivo Proof of Principle}

After 12 weeks of products application, the crow's feet wrinkles were significantly improved versus baseline with retinol $0.04 \%(\mathrm{p}=0.015)$, retinol $0.1 \%\left(\mathrm{p}=6 \times 10^{-5}\right)$, retinol $0.04 \%+$ DMC $0.1 \%(\mathrm{p}=0.00012)$. The addition of DMC to retinol $0.04 \%$ resulted in a significantly better efficacy compared to retinol $0.04 \%$ and similar to the efficacy of the product containing retinol 0.1\% (Table 2).

\subsection{Clinical Study Results}

\subsubsection{During the Application Period}

Wrinkles and fine lines were significantly improved with the active product versus baseline, the score differences varying from 0.23 to 1.03 (on a 10-point scale), after 4 and 8 weeks with p-values from $1.86 \times 10^{-3}$ to $6.74 \times$ $10^{-10}$, while no significant improvement of wrinkles was observed with the placebo. Moreover, the effect of the

Table 1. (a) Effect of DMC on collagen synthesis in human dermal fibroblasts culture. Collagen synthesis was measured by the incorporation of tritiated proline in the extracellular collagenic fraction. Results are expressed as dpm in collagenic fraction per 1000 cells; (b) Effect of the association retinol and DMC on the expression of retinoic activity marker in keratinocytes cultures. The expression of HB-EGF and CRABPII genes were measured after 48 hours of treatment by real time quantitative PCR.

(a)

\begin{tabular}{cc}
\hline Test product & Collagen synthesis. \\
\hline Control & $34.7 \pm 4.1$ \\
$\mathrm{TGF} \beta 1 \mathrm{ng} / \mathrm{ml}$ & $49.5 \pm 0.3^{*}$ \\
$\mathrm{DMC} 0.1 \mu \mathrm{g} / \mathrm{ml}$ & $46 \pm 2.2^{*}$ \\
$\mathrm{DMC} 1 \mu \mathrm{g} / \mathrm{ml}$ & $46.2 \pm 5.8^{*}$ \\
\hline
\end{tabular}

${ }^{*}$ Significant difference in $t$-test $\mathrm{p}<0.05$.

(b)

\begin{tabular}{ccc}
\hline Test product & $\begin{array}{c}\text { Expression of CRABP2 } \\
\text { mRNA (\% versus control) }\end{array}$ & $\begin{array}{c}\text { Expression of HB-EGF } \\
\text { mRNA \% versus control }\end{array}$ \\
\hline Control & $100 \pm 5$ & $100 \pm 10$ \\
Retinol 0.04\% & $325 \pm 16$ & $188 \pm 3$ \\
+DMC 0.1\% & $440 \pm 40$ & $268 \pm 35$ \\
Retinol 0.1\% & $561 \pm 86$ & $436 \pm 29$ \\
+ DMC 0.1\% & $747 \pm 144$ & $791 \pm 116$ \\
\hline
\end{tabular}

All results showed significant difference in $t$-test $\mathrm{p}<0.05 \mathrm{vs}$. control. 
Table 2. In vivo proof of principle: Clinical grading of the crow's feet wrinkles using a $12 \mathrm{~cm}$ visual analog scale.

\begin{tabular}{cccc}
\hline Test product & $\begin{array}{c}\text { Retinol } \\
0.1 \%\end{array}$ & $\begin{array}{c}\text { Retinol } \\
0.04 \%\end{array}$ & $\begin{array}{c}\text { Retinol 0.04\%+ } \\
\text { DMC 0.1\% }\end{array}$ \\
\hline Baseline & $3.1(0.5)$ & $2.7(0.4)$ & $3.9(0.5)$ \\
Week 12 & $\mathbf{1 . 9}(0.3)$ & $\mathbf{2 . 1}(0.3)$ & $\mathbf{2 . 2}(0.3)^{*}$ \\
Difference & 1.2 & 0.6 & 1.7 \\
\hline
\end{tabular}

These results are expressed as arithmetic means and standard deviation. Values in bold indicate significant improvement compared to baseline $(\mathrm{p}<$ 0.05 ). ${ }^{*}$ Difference between retinol $0.04 \%+$ DMC $0.1 \%$ and retinol $0.04 \%$ is significant $(\mathrm{p}<0.05)$

active product on wrinkles and fine lines was significantly higher relative to that of the placebo, as soon as 4 weeks, with $\mathrm{p}$-values from $\mathrm{p}=8.53 \times 10^{-3}$ to $\mathrm{p}=6.6 \times$ $10^{-15}$. Parameters related to skin tone and texture were also significantly improved versus both baseline and placebo.

Specifically, the assessment of brown spots showed score differences relative to $\mathrm{T} 0$ of 1.10 and 1.34 at $\mathrm{T} 4$ and $\mathrm{T} 8$, respectively. Radiance was improved by 1.87 and 2.05 after $\mathrm{T} 4$ and $\mathrm{T} 8$, while evenness skin tone increased from $\mathrm{T} 0$ by 1.3 and 1.55 at $\mathrm{T} 4$ and $\mathrm{T} 8$, respectively. All results were highly significant with $\mathrm{p}$ values varying from $1.12 \times 10^{-8}$ to $6.66 \times 10^{-9}$ (Figure 1).

Finally, grading of the overall photo-damage showed also a significant improvement with change in score values versus baseline of 0.86 after 4 weeks and 0.97 after 8 weeks $\left(\mathrm{p}<10^{-6}\right)$. These improvements were significantly higher than those obtained with the placebo (Table 3).

\subsubsection{At the End of the Regression Period}

Clinical grading was also performed 4 weeks after the end of the treatment with the active product. Results showed that 4 weeks after stopping the product application, improvement versus T0 was significantly maintained for crow's feet fine lines (1.12), crow's feet wrinkles (0.64), forehead wrinkles $(0.66)$, under eye wrinkles $(0.46)$ and cheek wrinkles $(0.51)$ for panelists that used the active product, with p-values between $5 \times 10^{-13}$ and $2.9 \times 10^{-5}$. Only crow's feet fine lines improvement was significantly maintained with the placebo $(\mathrm{p}=3.91 \times$ $10^{-2}$ ).

The differences between the efficacy of the active and placebo at the end of the regression period were still significant $\left(\mathrm{p}<10^{-5}\right)$ for parameters such as crow's feet fine lines, crow's feet wrinkles, forehead wrinkles, under eye wrinkles and cheek wrinkles (Figure 2).

The differences of the active product efficacy between $\mathrm{T} 0$ and T12 on the other signs of skin aging, such as brown spots (1.38), overall photodamage (1.11), radiance (2.38), and evenness skin tone (1.74) were still significantly improved, with $\mathrm{p}$-values inferior to $10^{-7}$. When the
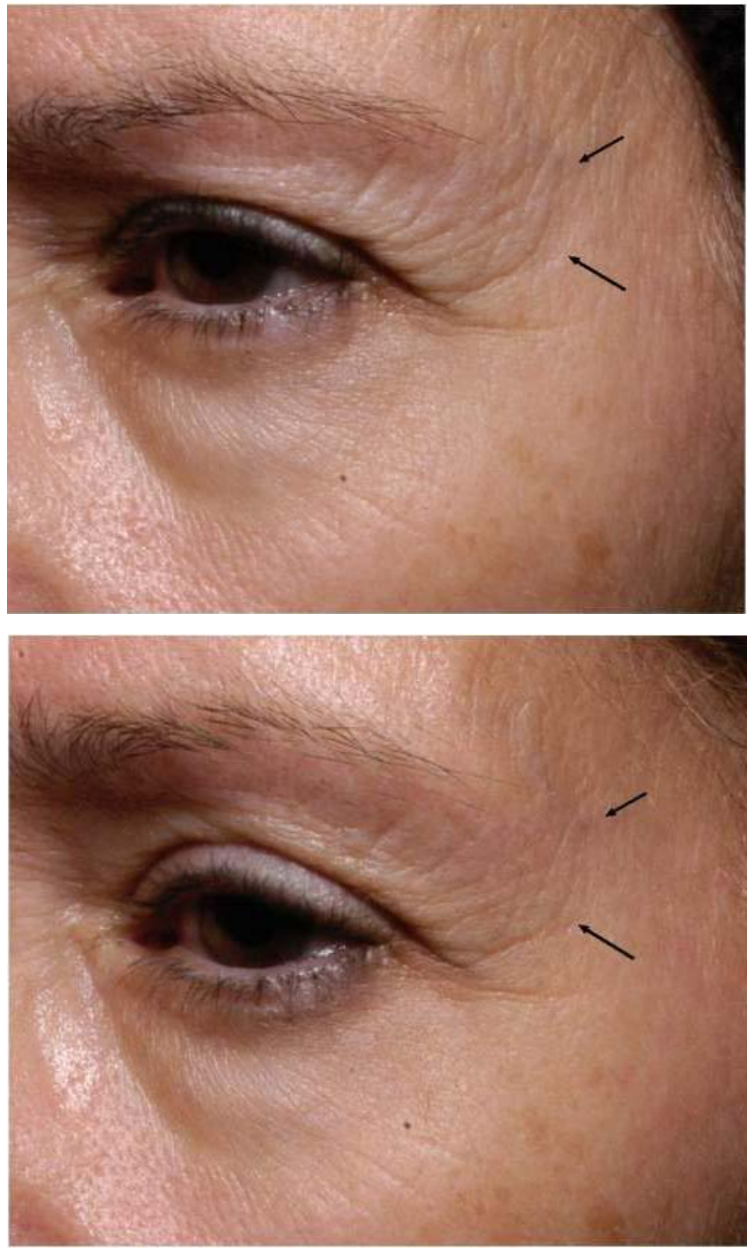

Figure 1. Pictures of eye area (crow's feet area) at baseline (top) and after 8 weeks (bottom).

placebo was used, the overall photodamage, radiance and evenness skin tone were also significantly improved. However, significant differences between active and placebo efficacy were still noticed on brown spots $(\mathrm{p}=3.06 \times$ $\left.10^{-12}\right)$, overall photodamage $\left(\mathrm{p}=1.39 \times 10^{-10}\right)$, skin radiance $\left(\mathrm{p}=4.34 \times 10^{-13}\right)$ and evenness skin tone $(\mathrm{p}=$ $\left.2.49 \times 10^{-7}\right)$ (Table 3).

\section{Discussion}

Retinoids are a family of compounds structurally related to retinol (vitamin A). They play an important role on epithelium proliferation and differentiation as well as exerting effects on the dermal matrix. Different studies have demonstrated the efficacy of topical tretinoin to improve photodamage. However, its use as topical treatment is complicated due to its irritation potential. On the other hand, retinol shows potential to reduce the signs of facial aging but with a much better tolerance profile. Recently Kafi et al. [32] demonstrated that topical applica- 
Table 3. In vivo clinical grading by the investigator of the other signs of ageing on 10-points scales (from $0=$ none to 9 = severe).

\begin{tabular}{|c|c|c|c|c|}
\hline Time (weeks) & $\begin{array}{l}\text { Brown } \\
\text { spots }\end{array}$ & $\begin{array}{c}\text { Overall } \\
\text { photo damage }\end{array}$ & $\begin{array}{c}\text { Skin } \\
\text { radiance }\end{array}$ & $\begin{array}{l}\text { Evenness } \\
\text { skin tone }\end{array}$ \\
\hline \multicolumn{5}{|l|}{ T0 } \\
\hline Active $(n=44)$ & $3.5(0.2)$ & $5.1(0.1)$ & $4.7(0.1)$ & $4.3(0.1)$ \\
\hline Placebo $(n=43)$ & $3.1(0.2)$ & $4.9(0.1)$ & $4.5(0.1)$ & $4.3(0.1)$ \\
\hline \multicolumn{5}{|l|}{$\mathrm{T} 4$} \\
\hline Active $(n=43)$ & $2.4(0.2)^{*}$ & $4.2(0.1)^{*}$ & $2.8(0.1)^{*}$ & $3.0(0.1)^{*}$ \\
\hline Placebo $(n=42)$ & $3.1(0.2)$ & $4.8(0.1)$ & $3.8(0.1)$ & $3.8(0.1)$ \\
\hline \multicolumn{5}{|l|}{$\mathrm{T} 8$} \\
\hline Active $(n=43)$ & $2.2(0.2)^{*}$ & $4.1(0.1)^{*}$ & $2.7(0.1)^{*}$ & $2.7(0.1)$ \\
\hline Placebo $(n=42)$ & $3.1(0.2)$ & $4.7(0.1)$ & $3.7(0.1)$ & $3.7(0.1)$ \\
\hline \multicolumn{5}{|l|}{ T12 (regression) } \\
\hline Active $(n=43)$ & $2.1(0.2)^{*}$ & $4.0(0.1)^{*}$ & $2.3(0.1)^{*}$ & $2.5(0.1)^{*}$ \\
\hline Placebo $(\mathrm{n}=42)$ & $3.1(0.2)$ & $4.6(0.1)$ & $3.5(0.1)$ & $3.5(0.1)$ \\
\hline
\end{tabular}

These results are expressed as arithmetic means and Standard Error Mean (SEM). Values in bold indicate significant improvement compared to T0 (baseline); $\mathrm{p}<0.05$. ${ }^{*}$ Difference between active and placebo is significant ( $\mathrm{p}$ $<0.05)$.

tion of a high concentration of retinol $(0.4 \%)$ on the upper inner arm of old volunteers reduces the signs of chronological aging. In the past, we have demonstrated that lower concentration of retinol $(0.1 \%)$ also delivers beneficial result on skin photoaging signs [33,34]. Even more, in the study described in this paper, we have demonstrated that the clinical efficacy of retinol could be achieved with a concentration of retinol as low as $0.04 \%$ by using it in combination with a chromone derivative and low molecular weight fragments of hyaluronic acid. The complementary effect between retinol and the DMC was confirmed in a small scale clinical study in which the association of retinol and DMC delivered a better clinical efficacy on wrinkles than retinol alone. It has to be noted that DMC tested alone at the same concentration $(0.1 \%)$ in the same formula did not deliver any improvement on wrinkles (data not shown). Ex vivo, topical application of retinol on skin has induced both keratinocyte proliferation and accumulation of extracellular matrix components such as pro-collagen and glucoaminoglycan [32]. We have previously showed that improvement of fine lines by retinol was correlated to its capacity to increase cell proliferation in the epidermis. It was not the case with the deep wrinkle suggesting that the wrinkle improvement by retinol might come from an effect on the extracellular matrix. Therefore, it can be assumed that the effect of DMC on retinol induced im-

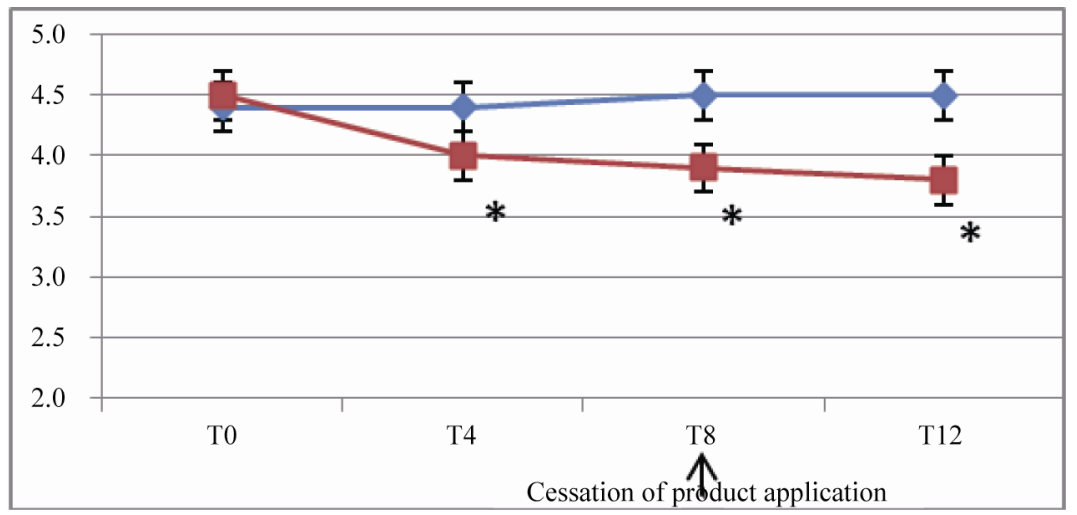

(a)

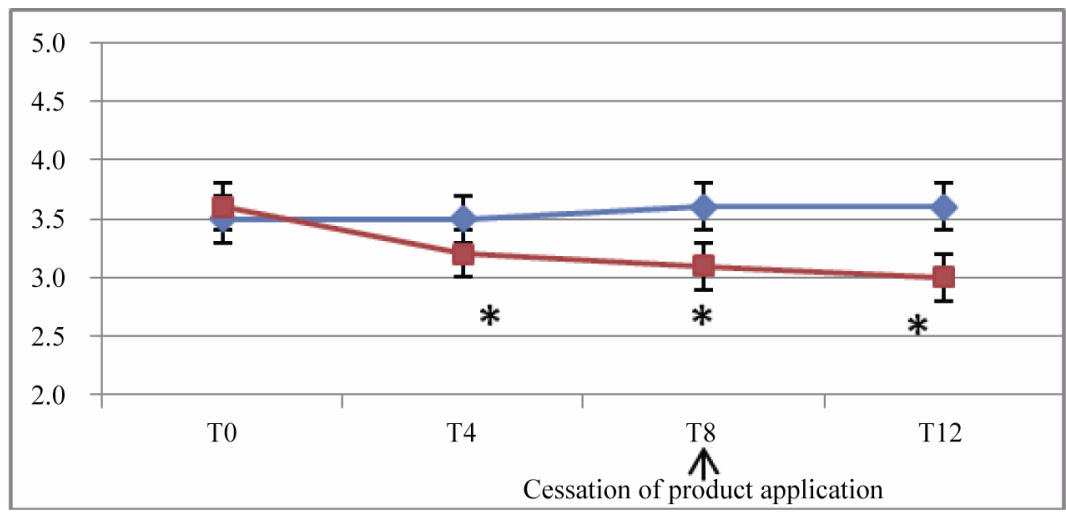

(b) 


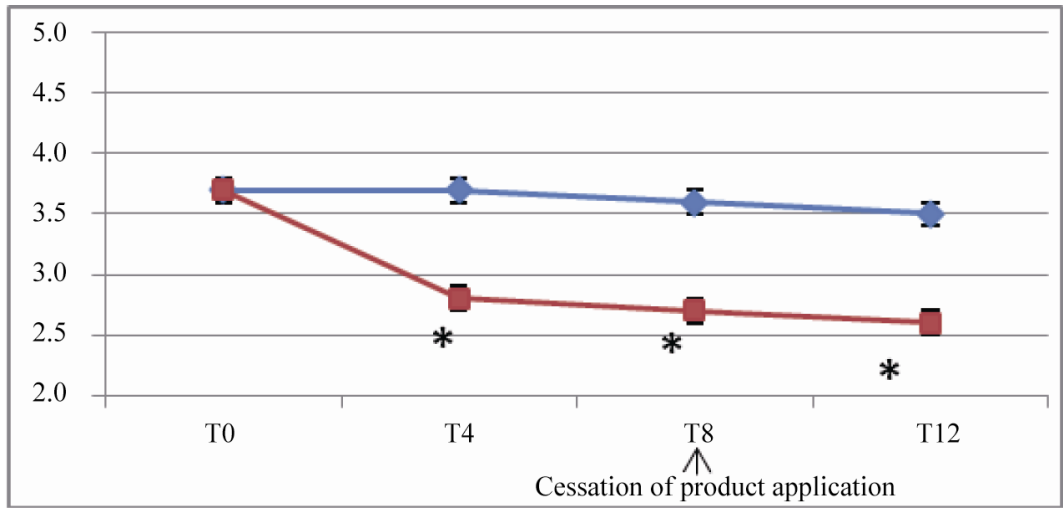

(c)

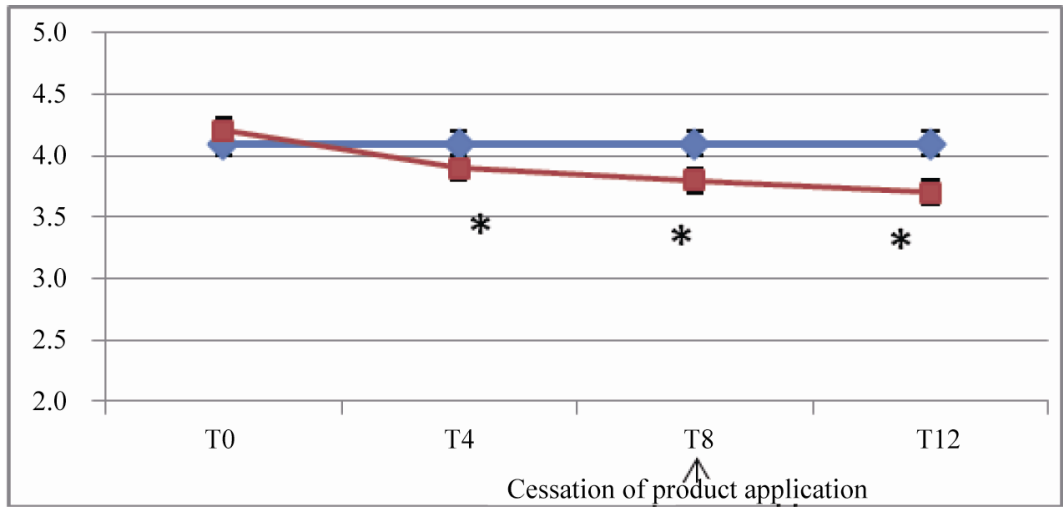

(d)

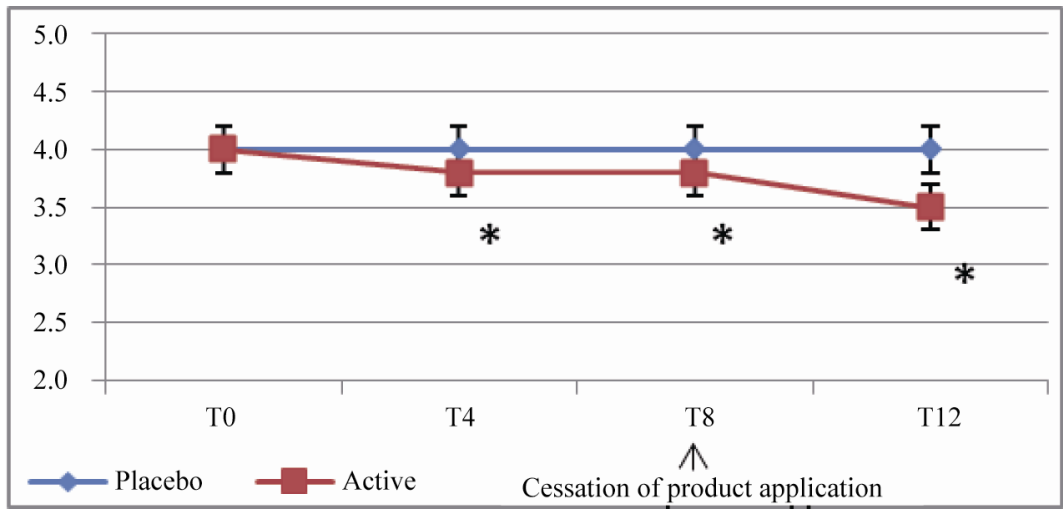

(e)

These results are expressed as arithmetic means. A star $\left(^{*}\right)$ indicates a significant difference compared to both T0 (baseline) and placebo; $\mathrm{p}<0.05$.

Figure 2. In vivo clinical grading by the investigator of the wrinkles and fine lines on 10-points scales (from $0=$ none to $9=$ severe). (a) Evolution of the score for forehead wrinkles; (b) Evolution of the score for crow's feet wrinkles; (c) Evolution of the score for crow's feet fine lines; (d) Evolution of the score for under eye wrinkles; (e) Evolution of the score for cheek wrinkles.

provement of wrinkle could be mediated by its capacity to trigger pro-collagen synthesis in dermal fibroblast and thus compensate the lower effect of low concentration of retinol in our product. In the clinical study, we demonstrated the potential combining retinol and DMC, in association with low molecular weight hyaluronic acid, to deliver in 8 weeks significant improvement on different aging signs such as wrinkles, brown spots, radiance and overall photoaging in 8 weeks of application.

Hyaluronic acid is described usually as a long polymer of several hundred thousand of kilodaltons (kd) that plays a role as a natural skin moisturizer in the epidermis 
through its capacity to bind and structure water. Several publications demonstrate that fragments of HA could also play a role in the epidermal physiology, by binding to specific receptors such as CD44, expressed on keratinocyte, and that topical use of HA fragments of around 50 - $400 \mathrm{kd}$ could reverse epidermal atrophy in mice [35]. Also, it has been demonstrated recently that low molecular weight HA fragments of $50 \mathrm{kd}$ could be delivered in reconstructed epidermis, and induced expression of different genes implicated in the terminal differentiation of keratinocytes, such as claudin and occludin genes [36]. Thus, low molecular weight fragments of HA, contained in our product, could also complement the effect of retinol on the keratinocytes, and complement its action to deliver improvement of skin aging signs, such as fine lines and radiance. The placebo significantly delivered benefits on skin tone and radiance, and, by consequence, on overall photodamage. This effect is the consequence of a moisturizing effect. Most of the parameters were more improved after 8 weeks compared to 4 weeks of application showing a certain degree of cumulative beneficial effect over time, with active product. More importantly, the improvement of the parameters brought by product was still significant 4 weeks after the cessation of the treatment. This result demonstrates that pursuing the application of a classical moisturizer, after the treatment period, is sufficient for maintaining the same level of active-induced benefits, even after a significant period of time, such as one month.

\section{Acknowledgements}

This study was totally funded by Johnson \& Johnson Santé Beauté France, Issy-les-Moulineaux, France.

\section{REFERENCES}

[1] S. Akiba, R. Shinkura, K. Miyamoto, G. Hillebrand, N. Yamaguchi and M. Ichihashi, "Influence of Chronic UV Exposure and Lifestyle on Facial Skin Photo-AgingResults from a Pilot Study," Journal of Epidemiology, Vol. 9, No. 6, 1999, pp. 136-142. doi:10.2188/jea.9.6sup 136

[2] G. Hillebrand, "Quantitative Evaluation of Skin Condition in an Epidemiological Survey of Females Living in Northern Versus Southern Japan," Journal of Dermatological Science, Vol. 27, No. 1, 2001, pp. 42-52. doi:10.1016/S0923-1811(01)00118-9

[3] S. Nouveau-Richard, Z. Yang, S. Mac-Mary, L. Li, P. Bastien, I. Tardy, et al., "Skin Ageing: A Comparison between Chinese and European Populations," Journal of Dermatological Science, Vol. 40, No. 3, 2005, pp. 187193. doi:10.1016/j.jdermsci.2005.06.006

[4] L. Petit, L. Fogouang, I. Uhoda, S. Smitz, C. PiérardFranchimont and G. Piérard, "Regional Variability in Mottled Subclinical Melanoderma in the Elderly," Ex- perimental Gerontology, Vol. 38, No. 3, 2003, pp. 327 331. doi:10.1016/S0531-5565(02)00200-0

[5] G. Fisher, S. Datta, Z. Wang, X. Li, T. Quan, J. Chung, et al., "c-Jun-Dependent Inhibition of Cutaneous Procollagen Transcription Following Ultraviolet Irradiation Is Reversed by All-trans Retinoic Acid," Journal of Clinical Investigation, Vol. 106, No. 5, 2000, pp. 663-670. doi:10.1172/JCI9362

[6] B. Gilchrest and M. Yaar, "Ageing and Photoageing of the Skin: Observations at the Cellular and Molecular Level," The British Journal of Dermatology, Vol. 127, No. 41, 1992, pp. 25-30. doi:10.1111/j.1365-2133.1992.tb16984.x

[7] W. Leung and I. Harvey, "Is Skin Ageing in the Elderly Caused by Sun Exposure or Smoking?" British Journal of Dermatology, Vol. 147, No. 6, 2002, pp. 1187-1191. doi:10.1046/j.1365-2133.2002.04991.x

[8] T. Quan, T. He, S. Kang, J. Voorhees and G. Fisher, "Solar Ultraviolet Irradiation Reduces Collagen in Photoaged Human Skin by Blocking Transforming Growth Factor- $\beta$ Type II Receptor/Smad Signaling," American Journal of Pathology, Vol. 165, No. 3, 2004, pp. 741-751. doi:10.1016/S0002-9440(10)63337-8

[9] H. Talwar, C. Griffiths, G. Fisher, T. Hamilton and J. Voorhees, "Reduced Type I and Type III Procollagens in Photodamaged Adult Human Skin," Journal of Investigative Dermatology, Vol. 105, 1995, pp. 285-290. doi:10.1111/1523-1747.ep12318471

[10] M. Brennan, H. Bhatti, K. Nerusu, N. Bhagavathula, S. Kang, G. Fisher, et al., "Matrix Metalloproteinase-1 Is the Major Collagenolytic Enzyme Responsible for Collagen Damage in UV-Irradiated Human Skin," Photochemistry and Photobiology, Vol. 78, No. 1, 2003, pp. 43-48. doi:10.1562/0031-8655(2003)0780043MMITMC2.0.CO2

[11] G. Fisher, S. Datta, H. Talwar, Z. Wang, J. Varani, S. Kang, et al., "Molecular Basis of Sun-Induced Premature Skin Ageing and Retinoid Antagonism," Nature, Vol. 379, 1996, pp. 335-339. doi:10.1038/379335a0

[12] V. Koivukangas, M. Kallioinen, H. Autio-Harmainen and A. Oikarinen, "UV Irradiation Induces the Expression of Gelatinases in Human Skin in Vivo," Acta DermatoVenereologica, Vol. 74, 1994, pp. 279-282.

[13] W. Montagna, S. Kirchner and K. Carlisle, "Histology of Sun-Damaged Human Skin," Journal of the American Academy of Dermatology, Vol. 21, No. 5, 1989, pp. 907918. doi:10.1016/S0190-9622(89)70276-0

[14] J. Seo, S. Lee, C. Youn, H. Choi, G. Rhie, K. Cho, et al., "Ultraviolet Radiation Increases Tropoelastin mRNA Expression in the Epidermis of Human Skin in Vivo," Journal of Investigative Dermatology, Vol. 116, 2001, pp. 915-919. doi:10.1046/j.1523-1747.2001.01358.x

[15] R. Warren, V. Gartstein, A. Kligman, W. Montagna, R. Allendorf and G. Ridder, "Age, Sunlight, and Facial Skin: A Histologic and Quantitative Study," Journal of the American Academy of Dermatology, Vol. 25, No. 5, 1991, pp. 751-760. doi:10.1016/S0190-9622(08)80964-4

[16] L. Baumann, "Skin Ageing and Its Treatment," Journal of Pathology, Vol. 211, No. 2, 2007, pp. 241-251. 


\section{doi:10.1002/path.2098}

[17] J. Leyden, "Clinical Features of Ageing Skin," British Journal of Dermatology, Vol. 122, No. 35, 1990, pp. 1-3. doi:10.1111/j.1365-2133.1990.tb16118.x

[18] J. Vitto, "Connective Tissue Biochemistry of the Aging Dermis: Age-Related Alterations in Collagen and Elastic," Dermatologic Clinics, Vol. 4, No. 3, 1986, pp. 433446.

[19] S. Cho, L. Lowe, T. Hamilton, G. Fisher, J. Voorhees and S. Kang, "Long-Term Treatment of Photoaged Human Skin with Topical Retinoic Acid Improves Epidermal Cell Atypia and Thickens the Collagen Band in Papillary Dermis," Journal of the American Academy of Dermatology, Vol. 53, No. 5, 2005, pp. 769-774. doi:10.1016/j.jaad.2005.06.052

[20] C. Griffiths, A. Russman, G. Majmudar, R. Singer, T. Hamilton and J. Voorhees, "Restoration of Collagen Formation in Photodamaged Human Skin by Tretinoin (Retinoic Acid)," New England Journal of Medicine, Vol. 329, 1993, p. 530. doi:10.1056/NEJM199308193290803

[21] G. Grove, M. Grove, J. Leyden, L. Lufrano, B. Schwab, B. Perry, et al., "Skin Replica Analysis of Photodamaged Skin after Therapy with Tretinoin Emollient Cream," Journal of the American Academy of Dermatology, Vol. 25, No. 2, 1991, pp. 231-237. doi:10.1016/0190-9622(91)70187-7

[22] H. Kim, N. Kim, S. Jung, J. Mun, J. Kim, B. Kim, et al., "Improvement in Skin Wrinkles from the Use of Photostable Retinyl Retinoate: A Randomized Controlled Trial," The British Journal of Dermatology, Vol. 162, No. 3, 2009, pp. 497-502.

[23] B. Chapellier, M. Mark, N. Messaddeq, C. Calleja, X. Warot, J. Brocard, et al., "Physiological and RetinoidInduced Proliferations of Epidermis Basal Keratinocytes Are Differently Controlled," The EMBO Journal, Vol. 21, 2002, pp. 3402-3413. doi:10.1093/emboj/cdf331

[24] S. Kang, E. Duell, G. Fisher, S. Datta, Z. Wang, A. Reddy, et al., "Application of Retinol to Human Skin in Vivo Induces Epidermal Hyperplasia and Cellular Retinoid Binding Proteins Characteristic of Retinoic Acid but without Measurable Retinoic Acid Levels or Irritation," Journal of Investigative Dermatology, Vol. 105, 1995, pp. 549-556. doi:10.1111/1523-1747.ep12323445

[25] L. Rittié, J. Varani, S. Kang, J. Voorhees and G. Fisher, "Retinoid-Induced Epidermal Hyperplasia Is Mediated by Epidermal Growth Factor Receptor Activation via Specific Induction of Its Ligands Heparin-Binding EGF and Amphiregulin in Human Skin in Vivo," Journal of Investigative Dermatology, Vol. 126, 2006, pp. 732-739. doi:10.1038/sj.jid.5700202

[26] J. Varani, R. Warner, M. Gharaee-Kermani, S. Phan, S. Kang, J. Chung, et al., "Vitamin A Antagonizes Decreased Cell Growth and Elevated Collagen-Degrading
Matrix Metalloproteinases and Stimulates Collagen Accumulation in Naturally Aged Human Skin," Journal of Investigative Dermatology, Vol. 114, 2000, pp. 480-486. doi:10.1046/j.1523-1747.2000.00902.x

[27] I. Effendy, S. Weltfriend, S. Patil and H. Maibach, "Differential Irritant Skin Responses to Topical Retinoic Acid and Sodium Lauryl Sulphate: Alone and in Crossover Design," British Journal of Dermatology, Vol. 134, No. 3, 1996, pp. 424-430. doi:10.1046/j.1365-2133.1996.26761.x

[28] V. Goffin, F. Henry, C. Piérard-Franchimont and G. Piérard, "Topical Retinol and the Stratum Corneum Response to an Environmental Threat," Skin Pharmacology, Vol. 10, No. 2, 1997, pp. 85-89. doi:10.1159/000211473

[29] B. Kim, Y. Lee and K. Kang, "The Mechanism of Retinol-Induced Irritation and Its Application to Anti-Irritant Development," Toxicology Letters, Vol. 146, No. 1, 2003, pp. 65-73. doi:10.1016/j.toxlet.2003.09.001

[30] E. Calikoglu, O. Sorg, C. Tran, D. Grand, P. Carraux, J. Saurat, et al., "UVA and UVB Decrease the Expression of CD44 and Hyaluronate in Mouse Epidermis, Which Is Counteracted by Topical Retinoids," Photochemistry and Photobiology, Vol. 82, No. 5, 2006, pp. 1342-1347. doi:10.1562/2006-02-10-RA-801

[31] G. Kaya, D. Grand, R. Hotz, E. Augsburger, P. Carraux, L. Didierjean, et al., "Upregulation of CD44 and Hyaluronate Synthases by Topical Retinoids in Mouse Skin," Journal of Investigative Dermatology, Vol. 124, 2005, pp. 284-287. doi:10.1111/j.0022-202X.2004.23579.x

[32] R. Kafi, H. Kwak, W. Schumacher, S. Cho, V. Hanft, T. Hamilton, et al., "Improvement of Naturally Aged Skin With Vitamin A (Retinol)," Archives of Dermatology, Vol. 143, 2007, p. 606. doi:10.1001/archderm.143.5.606

[33] G. Bellemere, G. N. Stamatas, V. Bruere, C. Bertin, N. Issachar and T. Oddos, "Antiaging Action of Retinol: from Molecular to Clinical," Skin Pharmacology and Physiology, Vol. 22, No. 4, 2009, pp. 200-209. doi: $10.1159 / 000231525$

[34] S. Tucker-Samaras, T. Zedayko, C. Cole, D. Miller, W. Wallo and J. Leyden, "A Stabilized 0.1\% Retinol Facial Moisturizer Improves the Appearance of Photodamaged Skin in an Eight-Week, Double-Blind, Vehicle-Controlled Study," Journal of Drugs in Dermatology, Vol. 8, 2009, p. 932.

[35] G. Kaya, C. Tran, O. Sorg, R. Hotz, D. Grand, P. Carraux, et al., "Hyaluronate Fragments Reverse Skin Atrophy by a CD44-Dependent Mechanism," PLoS Medicine, Vol. 3, 2006, p. 493. doi:10.1371/journal.pmed.0030493

[36] M. Farwick, G. Gauglitz, T. Pavicic, T. Köhler, M. Wegmann, K. Schwach-Abdellaoui, et al., "Fifty-kDa Hyaluronic Acid Upregulates Some Epidermal Genes without Changing TNF- $\alpha$ Expression in Reconstituted Epidermis," Skin Pharmacology and Physiology, Vol. 24, No. 4, 2011, pp. 210-221. doi:10.1159/000324296 